\title{
Chemical Potential Driven Reorganization of Anions between Stern and Diffuse Layers at the Air/Water Interface
}

\author{
Raju R. Kumal, ${ }^{1}$ Srikanth Nayak, ${ }^{1}$ Wei Bu, ${ }^{2}$ and Ahmet Uysal ${ }^{l, ~ *}$
}

${ }^{1}$ Chemical Sciences and Engineering Division, Argonne National Laboratory, Lemont, IL 60439, United States

${ }^{2}$ NSF's ChemMatCARS, The University of Chicago, Chicago, Illinois 60637, United States

KEYWORDS: Liquid-liquid extraction (LLE), vibrational sum frequency generation (VSFG),

selenocyanate, interface, X-ray reflectivity (XR), X-ray fluorescence near total reflection

(XFNTR)

TOC Graphic

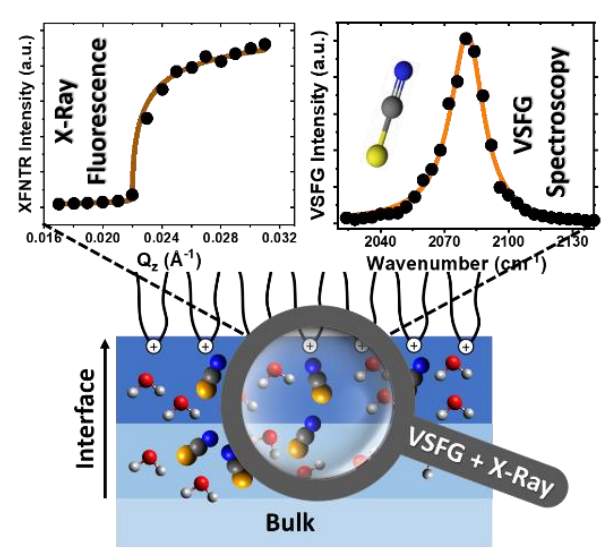




\begin{abstract}
Ion adsorption and transfer at charged interfaces play key roles in various industrial and environmental processes. Molecular scale details of ion-ion, ion-water, and ion-surface interactions and their dependence on the character of the ion (ion-specific effects) are still debated. Complex ions, such as $\mathrm{SCN}^{-}$and $\mathrm{SeCN}^{-}$, are particularly interesting due to their unexpected adsorption trends which are ascribed to their permanent dipole moment and non-spherical shape. Here, we combine vibrational sum frequency generation (VSFG) spectroscopy and surface sensitive synchrotron $\mathrm{X}$-ray studies to provide a detailed description of $\mathrm{SeCN}^{-}$adsorption at a charged surfactant monolayer at the air/aqueous interface. Using multiple polarization combinations in VSFG to probe the $-\mathrm{CN}$ stretch, we show that the average orientation of $\mathrm{SeCN}^{-}$ anions with respect to the surface normal changes from $45^{\circ}$ to $22^{\circ}$ with the increasing $\mathrm{KSeCN}$ concentration. X-ray fluorescence experiments of Se K-edge and VSFG studies of -OH stretch show that $\mathrm{SeCN}^{-}$interfacial coverage (Stern layer + diffuse layer) saturates at very low bulk concentrations, but their orientational organization, distribution between Stern and diffuse layers, and effects on the hydrogen bonding network of the interfacial water continue to change with increasing bulk KSeCN concentration. X-ray reflectivity (XR) studies provide further evidence for increasing $\mathrm{SeCN}^{-}$population in Stern layer correlated with the orientational reorganization. These results show that the increasing chemical potential may lead to further reorganization of the adsorbed ions, even though the total interfacial ion population does not change. The reorganization of the interfacial ions and the water may be very important in chemical separations of heavy metals, where metal-anion complexes drive the selective ion transfer at aqueous interfaces.
\end{abstract}




\section{INTRODUCTION}

Aqueous interfaces and how ions behave near them control many important processes, such as protein folding, ${ }^{1}$ ice nucleation, ${ }^{2,3}$ mineralization, ${ }^{4,5}$ drug delivery, ${ }^{6}$ and chemical separations. ${ }^{7-}$

${ }^{13}$ The common point of all these processes is that the molecular scale interface significantly differs from the bulk in terms of the ion concentration, the ion speciation, and the water structure. For instance, water is layered and orientationally ordered at interfaces, ${ }^{14,15}$ the dielectric constant is significantly lower $(\sim 5)$ than the bulk value $(\sim 80),{ }^{16,17}$ and the ionic species that are not present in the bulk can be stable at the interface. ${ }^{18}$ All these differences make it difficult to predict how ions behave at interfaces based on the bulk solution properties. The difficulty increases when the ion cannot be treated as a zero-dimensional unit charge interacting with an ideal surface. Its finite size, hydration enthalpy, polarizability, and bonding properties, which are usually considered in the context of ion-specific effects, as well as the nature of the surface functional groups may lead to complex adsorption trends. ${ }^{19-21}$

Ion-specific effects are usually considered in an empirical framework, where ions are listed in ascending or descending order with respect to a certain effect, such as their ability to salt out proteins as described in the Hofmeister series. ${ }^{19,20}$ It is not uncommon to see the certain ions switch positions on these empirical lists depending on the other factors in the system. In complex systems, where multiple enthalpic and entropic factors create an intricate free energy landscape, ion-specific effects may lead to qualitative differences in the ion adsorption and transfer mechanisms. For example, both nitrate $\left(\mathrm{NO}_{3}{ }^{-}\right)$and thiocyanate $\left(\mathrm{SCN}^{-}\right)$are classified as chaotropic anions in the Hofmeister series but they organize interfacial structure differently. ${ }^{22}$ Adsorption of $\mathrm{NO}_{3}{ }^{-}$does not change the interfacial water structure significantly with increasing concentration while $\mathrm{SCN}^{-}$ causes significant changes, resulting in the reorganization of the interfacial water. These nanoscale 
differences become very important in complex processes, such as chemical separations. For instance, in liquid-liquid extraction (LLE) of the rare earth metals, the extraction trends for heavy or light lanthanides are completely reversed in the presence of nitrate or thiocyanate.

Thiocyanate is one of the most studied pseudohalides due to its unique interfacial properties, and its applications in separation science and electrochemistry. It is a highly surfaceactive species with significant dipole moment $\left(\mu_{R-S C N}=2.99 \mathrm{D}\right){ }^{23}$ which most likely navigates the ion to favorable orientation at interfaces. Recent experimental and theoretical studies have demonstrated the existence of enhanced concentration of $\mathrm{SCN}^{-}$anions at the air/water interface even at very low bulk concentrations. ${ }^{24,25}$ Viswanath et al. and Bian et al. showed that the average tilt angle of $\mathrm{SCN}^{-}$anions is $45^{\circ}$ with respect to the surface normal at the free air/water interface. ${ }^{24}$, ${ }^{26-28}$ Saykally et al. have studied the thermodynamics of $\mathrm{SCN}^{-}$adsorption, and its charge-transferto-solvent spectrum at air/water interface and analyzed the solvation environments. ${ }^{29}, 30$ Selenocyanate $\left(\mathrm{SeCN}^{-}\right)$has similar properties to that of $\mathrm{SCN}^{-}$and have been used as a vibrational tag to investigate the solvent environments. ${ }^{31}$ The strongest $\mathrm{CN}$ stretch frequency mode occurs at $\sim 2070 \mathrm{~cm}^{-1}$ for $\mathrm{SCN}^{-}$and $\sim 2080 \mathrm{~cm}^{-1}$ for $\mathrm{SeCN}^{-} .22,32$ This small change is due to the slight variation in hydrogen bond strength leading to different transition dipole moments. ${ }^{33,34}$ The dipole moment of $\mathrm{SeCN}^{-}$is reported slightly higher than that of $\mathrm{SCN}^{-}\left(\mu_{R-S e C N}=3.48 D\right) \cdot{ }^{23}$ Most of these studies are focused on investigation of the local structures and dynamics of anions at aqueous interfaces, but there are several unanswered questions which yet to be addressed such as interfacial hydration and organization of ions in LLE.

It is important to understand the impact of ion-specific effects in complex chemical processes, such as LLE of heavy and precious metals. In LLE metal ions are selectively transferred from an aqueous matrix into an organic phase with the help of amphiphilic molecules (also called 
extractants). ${ }^{35}$ Since LLE is free energy driven at ambient conditions, it is relatively less energy intensive compared to other separation methods, such as distillation. The background anions in the aqueous phase have a significant impact on the extraction efficiency and selectivity. For example, heavy and light lanthanides are extracted better from $\mathrm{SCN}^{-}$and $\mathrm{NO}_{3}{ }^{-}$solutions, respectively, when quaternary amine methyltrioctyl ammonium (TOMA) is the extractant. ${ }^{36}$ Multiple factors, such as aqueous speciation of ions, interfacial interactions, nature of the diluent, and extractant-ion complexes formed in the organic phase are generally responsible for the selective transfer of the metal ions from an aqueous to an organic phase..$^{37,38}$

In separation science, it is well known that the interfacial region can affect the mass transfer and partitioning. ${ }^{39,40}$ However, it is poorly characterized due to the experimental inaccessibility of the interface in a real extraction system. Therefore, model interfacial systems consisting of air/liquid or liquid/liquid interfaces are used to understand the interfacial interactions between extractants and ions. In general, model systems having a Langmuir monolayer have been very effective to study the interfacial water structure, ${ }^{41,42}$ interaction of cations with monolayer, ${ }^{43,44}$ phase transfer of metal ions, ${ }^{11}$ and water hydrogen bond rearrangement dynamics. ${ }^{9,}{ }^{45}$ With the advent of surface specific techniques such as vibrational sum-frequency generation (VSFG) spectroscopy, second harmonic generation (SHG) spectroscopy, and synchrotron X-ray scattering measurements, it has become possible to differentiate the interfacial phenomena from the bulk and study the structure and the dynamics of interfaces at the molecular level. ${ }^{46-51}$

VSFG and X-ray scattering are sensitive to different aspects of the interface. Therefore, their combination provides a significantly better understanding that cannot be obtained from one of them alone. ${ }^{9,22,52,53}$ For instance, VSFG signal intensity from the $-\mathrm{CN}$ stretch is a combination of the number density and the orientational ordering of the $\mathrm{SeCN}^{-}$ions. X-ray fluorescence near 
total reflection (XFNTR) provides the interfacial number density directly. Combining these two techniques allow decoupling the contributions from the orientational ordering and the number density in VSFG experiments.

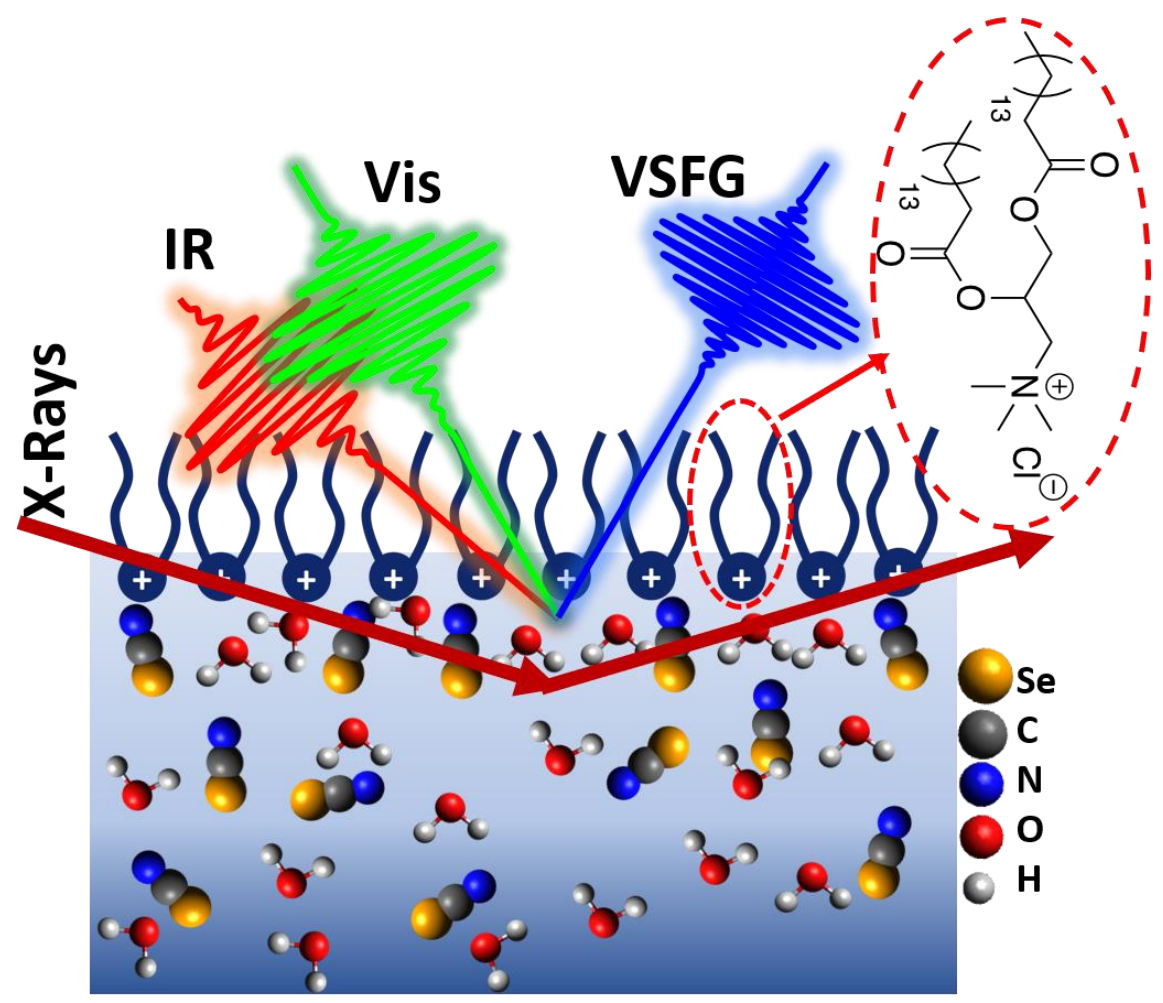

Figure 1. A schematic describing the VSFG and synchrotron X-ray experiments at the air/aqueous interface. The inset shows the structure of the DPTAP ${ }^{+}$surfactant.

We use a monolayer of $\mathrm{DPTAP}^{+}$to model the commonly used industrial extractant quaternary ammonium $\mathrm{TOMA}^{+}$. $\mathrm{DPTAP}^{+}$forms a stable monolayer at air/aqueous interface due to its longer hydrophobic carbon chain. It has been extensively studied to investigate the adsorption of ions at charged interfaces. ${ }^{22,53-57}$ The schematic representation of air/aqueous interface with $\mathrm{DPTAP}^{+}$monolayer, in the presence of $\mathrm{SeCN}^{-}$anions in the subphase, is shown in Figure 1. Tunable IR and fixed visible beams are overlapped spatially and temporally to generate VSFG signal from the interface. Because bulk molecules have an inversion symmetry, the VSFG signal 
originates purely from the interfacial region. ${ }^{58}$ The incoming X-ray beam is used to generate XFNTR and XR signals from the interface.

XFNTR is used to quantify the amount of $\mathrm{SeCN}^{-}$anions adsorbed to a charged DPTAP ${ }^{+}$ monolayer at air/aqueous interface. The incoming beam of X-rays does not penetrate into the bulk phase at small incidence angles due to total external reflection and only excites the interfacial ions. $^{4,59,60}$ This is a highly surface sensitive technique which can quantify the elemental composition at the interface depending on the element's X-ray absorption energy. In XFNTR, the incident beam is directed to the interface slightly below and above the critical angle and the collected fluorescence signal is plotted as a function of the incidence angle. Below the critical angle, only the interfacial ions contribute to the fluorescence signal. ${ }^{61,62}$ Above the critical angle, both interfacial and bulk ions contribute to the signal.

Here, we combine VSFG, XFNTR and XR measurements to investigate the $\mathrm{SeCN}^{-}$ adsorption at $\mathrm{DPTAP}^{+}$monolayer at the air/water interface. $\mathrm{SeCN}^{-}$is used because $\mathrm{Se} \mathrm{K}$-edge $(12.66 \mathrm{keV})$, is more convenient than $\mathrm{S}$ K-edge $(2.47 \mathrm{keV})$ for the XFNTR experiments. A comparison of XFNTR data to the VSFG signal from the $-\mathrm{CN}$ stretch under the same conditions showed that the $\mathrm{SeCN}^{-}$ions reorganize at the interface even after their interfacial number density is saturated as a function of the bulk concentration. VSFG studies of the -OH stretch of the water molecules and XR experiments support this interpretation and further elucidates that the reorganization is mainly due to the increasing $\mathrm{SeCN}^{-}$population in Stern layer in the expense of their decreasing population in diffuse layer.

\section{EXPERIMENTAL METHODS}

2a. Sample Preparation. Chloride salt of DPTAP was purchased from Avanti Polar Lipids and stored at $-20{ }^{\circ} \mathrm{C}$. Potassium selenocyanate ( $\left.\mathrm{KSeCN}, \geq 98 \%\right)$ and HPLC grade chloroform 
$\left(\mathrm{CHCl}_{3}, \geq 99.9 \%\right)$ were purchased from Sigma Aldrich. All glassware and sample cell used for the experiment were cleaned by soaking in a solution of Nochromix (GODAX Laboratories, Inc.) in concentrated sulfuric acid bath for 24 hours and rinsed with ultrapure water $(18.2 \mathrm{M} \Omega . \mathrm{cm})$.

For the preparation of Langmuir monolayer, $0.25 \mathrm{mM}$ DPTAP solution was made in chloroform and stored at $-20^{\circ} \mathrm{C}$ temperature. A circular polytetrafluoroethylene (PTFE) dish with $6 \mathrm{~cm}$ diameter was used as a sample cell for VSFG experiment. $0.25 \mathrm{mM}$ DPTAP solution was added dropwise using $1 \mu \mathrm{L}$ syringe (Hamilton, USA) in a sample cell containing $25 \mathrm{~mL}$ of subphase solution. A NIMA pressure sensor, with a chromatography paper as a Wilhelmy plate, was used to measure the surface pressure. All experiments were performed at a surface pressure of $10 \mathrm{mN} / \mathrm{m}$ at room temperature.

2b. VSFG Experiment. The VSFG measurements are acquired using an EKSPLA system, which has been described previously. ${ }^{9,22,63}$ Briefly, the setup consists of a picosecond laser system, a harmonic unit, an optical parametric generator with difference frequency generation, a spectrometer and a photomultiplier tube detector connected to a monochromator. An amplified Nd:YAG laser system produces $29 \mathrm{ps}$ pulses having $28 \mathrm{~mJ}$ energy centered at $1064 \mathrm{~nm}$ with a repetition rate of $50 \mathrm{~Hz}$. The harmonic unit splits the $1064 \mathrm{~nm}$ laser, and a portion is passed through a second harmonic crystal to generate two beams of $532 \mathrm{~nm}$. One of the $532 \mathrm{~nm}$ beams and the $1064 \mathrm{~nm}$ beam are used to generate a narrowband IR pulse tunable from $1000-4000 \mathrm{~cm}^{-1}$ via optical parametric generator and difference frequency generation. Other $532 \mathrm{~nm}$ laser beam which passes through an adjustable delay stage is overlapped spatially and temporarily with the IR beam to generate the sum frequency (SF) signal. The polarization of $532 \mathrm{~nm}$ is adjusted with a $\lambda / 2$ waveplate, and the IR polarization is adjusted by using computer-controlled motorized mirrors. 
The SFG signal polarization is selected using a Glan polarizer. The SFG signal is then directed to a monochromator and collected with a photomultiplier tube.

The VSFG spectrometer employs reflection geometry where the incident angles of the visible and IR beams are $60^{\circ}$ and $55^{\circ}$, respectively, to the surface normal. The visible light is attenuated to an average energy of $600 \mu \mathrm{J}$ and the IR energy is maintained at $100 \mu \mathrm{J}$ for all measurements. A motorized piezoelectric rotation stage is used to rotate the sample to avoid beam damage. Each spectrum is collected with a $4 \mathrm{~cm}^{-1}$ increment over the range of $2800-3800 \mathrm{~cm}^{-1}$ and averaged over 300 laser shots per point. The spectra are collected under various polarization combinations and are normalized against the SFG spectrum of a z-cut quartz.

Theory of VSFG Spectroscopy. Vibrational sum-frequency generation (VSFG) is a second order non-linear spectroscopic process which is widely used for the investigation of interfacial molecular structure and dynamics. In VSFG, two input visible and IR laser beams at frequencies $\omega_{\text {vis }}$ and $\omega_{\text {IR }}$ overlap spatially and temporally on a sample surface to generate a sum frequency $\left(\omega_{\mathrm{SF}}=\omega_{\mathrm{vis}}+\omega_{\mathrm{IR}}\right)$ signal. Due to quantum mechanical selection rule of VSFG spectroscopy, the process is forbidden in the centrosymmetric media and thus eliminates the contributions from bulk phase..$^{51,64,65}$ The intensity of the VSFG signal $\left(\mathrm{I}_{\mathrm{SF}}\right)$ is proportional to the product of the intensities of the incident beams and the square of the effective second order nonlinear susceptibility $\left(\chi_{e f f}^{(2)}\right)$ of the material interface. This can be expressed as; ${ }^{66-69}$

$$
I_{S F}=\frac{8 \pi^{3} \omega_{S F}^{2} \sec ^{2} \beta}{c^{3} n_{1}\left(\omega_{S F}\right) n_{1}\left(\omega_{\text {vis }}\right) n_{1}\left(\omega_{I R}\right)}\left|\chi_{\text {eff }}^{(2)}\right|^{2} I\left(\omega_{\text {vis }}\right) I\left(\omega_{\text {IR }}\right)
$$

where, $\beta$ is the reflection angle of the sum frequency field and $n_{1}$ is the refractive index of corresponding medium. There are 27 components of $\chi^{(2)}$ but in the case of azimuthally isotropic interface, there are only four independent non-vanishing components; $\chi_{x x z}=\chi_{y y z} ; \chi_{z x x}=$ 
$\chi_{z y y} ; \chi_{x z x}=\chi_{y z y} ;$ and $\chi_{z z z}$, where $\mathrm{z}$ is defined to be the direction normal to the surface. These four components can be deduced by measuring VSFG at different polarizations: SSP, SPS, PSS and PPP, (SF, visible, and IR fields, respectively). The $\chi_{\text {eff }}^{(2)}$ consists of non-resonant $\left(\chi_{N R}^{(2)}\right)$ and resonant $\left(\chi_{R}^{(2)}\right)$ terms. The VSFG intensity is enhanced when the frequency of incidence IR beam is on resonance with a vibrational mode of an interfacial molecule.

$$
\left|\chi_{e f f}^{(2)}\right|^{2} \propto\left|\chi_{N R}^{(2)}+\sum_{q} \frac{A_{q}}{\omega_{I R}-\omega_{q}+i \Gamma_{q}}\right|^{2}
$$

Where, $A_{q}$ is resonance amplitude, $\omega_{q}$ is resonant frequency, and $\Gamma_{q}$ is damping constant of $\mathrm{q}^{\text {th }}$ vibrational mode which describes the linewidth of the transition. The $\chi_{\text {eff }}^{(2)}$ term can also be expressed in the following form.

$$
\chi_{e f f}^{(2)}=N_{s} d\left(\langle\cos \theta\rangle-c\left\langle\cos ^{3} \theta\right\rangle\right)=N_{s} d r(\theta)
$$

where, $\mathrm{N}_{\mathrm{s}}$ is effective surface number density, $r(\theta)$ is orientational distribution, $\theta$ is the molecular orientation angle to the surface normal, $d$ is the susceptibility strength factor proportional to the hyperpolarizability value, and $\mathrm{c}$ is called the general orientational parameter.

2c. Synchrotron X-ray Experiments. XFNTR and XR are used for the investigation of interfacial population and organization of ions at air/aqueous interface. XR and XFNTR measurements were carried out using a Langmuir trough integrated with a synchrotron X-ray beamline at sector 15-ID-C, NSF's ChemMatCARS of the Advanced Photon Source at Argonne National Laboratory. X-ray energy was $17.3 \mathrm{keV}$ and all experiments were conducted at room temperature. The Langmuir trough was enclosed in a gas-tight chamber and was purged with helium to reduce the beam damage and the background scattering. XR signal was recorded by a Pilatus $100 \mathrm{~K}$ area detector and XNFTR signal was recorded by a Votex-60 EX multicathode energy dispersive X-ray detector placed $10 \mathrm{~mm}$ above from the sample surface. 
The XR data were measured as a function of the wave vector transfer, $\overrightarrow{Q_{z}}=(4 \pi / \lambda) \sin \alpha$ by varying the incidence angle $(\alpha)$. For $17.3 \mathrm{keV}$ energy, the wavelength $(\lambda)$ of the $\mathrm{X}$-ray beam is $0.72 \AA ̊$ A The DPTAP monolayer is modeled by two-slabs for $2.5 \mu \mathrm{M}$ and $25 \mathrm{mM}$ samples, with one slab corresponding to the hydrophobic tail group and other for the hydrophilic head group plus the adsorbed ions. The intermediate concentration, $2.5 \mathrm{mM}$, sample required a three-box model. The thickness, electron density, and the roughness of the layers are determined by fitting the experimental data using a Parratt algorithm. ${ }^{59,62,70}$ The X-ray energy of $17.3 \mathrm{keV}$ used for the XNFTR measurement was above the K absorption edge of Se (12.66 keV). 

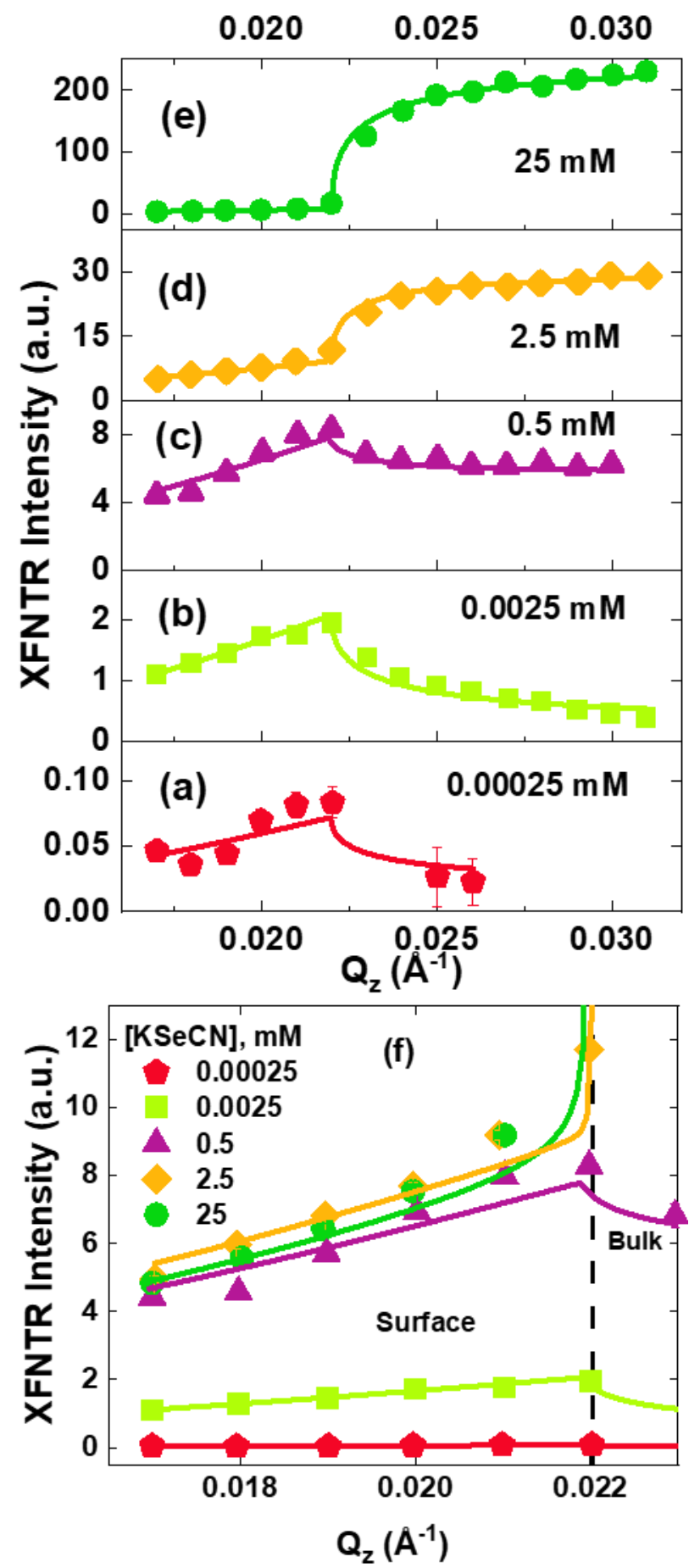

Figure 2. (a-e) XFNTR measurements at different $\mathrm{SeCN}^{-}$concentrations. The solid lines are the best fits to the experimental data. (f) Zoomed in version of the data and fit below the critical angle $\left(\mathrm{Q}_{\mathrm{c}} \sim 0.022 \AA^{-1}\right)$ for all data sets in a-e, displaying the change in XFNTR intensity due to $\mathrm{SeCN}^{-}$ adsorption at the interfacial region. 


\section{RESULTS AND DISCUSSION}

Figure 2 (a-e) show the variation of XFNTR intensity as a function of the vertical momentum transfer $\left(\mathrm{Q}_{\mathrm{z}}\right)$ for $\mathrm{SeCN}^{-}$adsorption at DPTAP monolayer at various bulk $\mathrm{KSeCN}$ concentrations. A similar measurement from a $25 \mathrm{mM} \mathrm{KSeCN}$ solution without a monolayer was used for calibration (Figure S1). The solid lines are the best fits to the experimental data. The fits use a model that considers the geometry of the experimental setup, and the X-ray absorption and emission energies. ${ }^{4,71}$ In the presence of the monolayer, the intensity of XFNTR signal increases linearly with the increasing $\mathrm{Q}_{z}$ below the critical angle $\left(\mathrm{Q}_{\mathrm{c}}=0.022 \AA^{-1}\right)$, indicating the anion adsorption at the interface. At low bulk concentrations, the signal intensity decreases above $\mathrm{Q}_{c}$, due to the decreasing transmission and very small bulk concentration. Whereas, for higher bulk concentrations, the bulk signal dominates above $\mathrm{Q}_{\mathrm{c}}$. The data below the critical angle is shown in Figure 2(f) separately to better display the changes in fluorescence intensity caused by the interfacial ion adsorption. The total number of $\mathrm{SeCN}^{-}$anions adsorbed at the interface is saturated at $0.5 \mathrm{mM}$ bulk concentration, which can be understood from the raw data without fitting (Figure 2f). The fit results show that the molecular area per $\mathrm{SeCN}^{-}$saturates at $\sim 53 \AA^{2}$, which is slightly more than the area per DPTAP ${ }^{+}$molecule for high concentration samples $\left(\sim 43 \AA^{2}\right.$, obtained from GID measurements, Figure S2) ${ }^{22}$ suggesting that the charge neutrality is nearly satisfied between the positively charged headgroups and the adsorbed $\mathrm{SeCN}^{-}$ions. XFNTR does not distinguish between Stern and diffuse layers and provides the total number of interfacial $\mathrm{SeCN}^{-}$ions. Below, we discuss how the orientational ordering of $\mathrm{SeCN}^{-}$ions continues to change even after this overall saturation.

The vibrational signatures of $\mathrm{CN}$ stretch region $\left(2000-2200 \mathrm{~cm}^{-1}\right)$ of $\mathrm{SeCN}^{-}$ions under PPP, SSP, and SPS polarization combinations are directly probed using VSFG spectroscopy. Figure 3 
(a-c) shows the variation of VSFG intensity as a function of IR frequency at various KSeCN concentrations under different polarization combinations. A single-peak Lorentz function (equation 2) is used to fit the experimental data under PPP and SPS polarizations and the fit parameters are tabulated in Table S1. The signal intensity under PPP polarization is higher and monotonically increases with increasing $\mathrm{KSeCN}$ bulk concentration. On the other hand, VSFG signal under SSP and SPS polarizations are found to be less sensitive to the bulk concentration. Under SSP polarization, the VSFG intensity is almost indistinguishable from the background and does not increase with increasing concentration. The same measurement under SPS polarization results slightly higher intensity compared to SSP case and appears to increase slightly for the lowest three $\mathrm{KSeCN}$ concentrations before plateauing at around $0.025 \mathrm{mM}$.
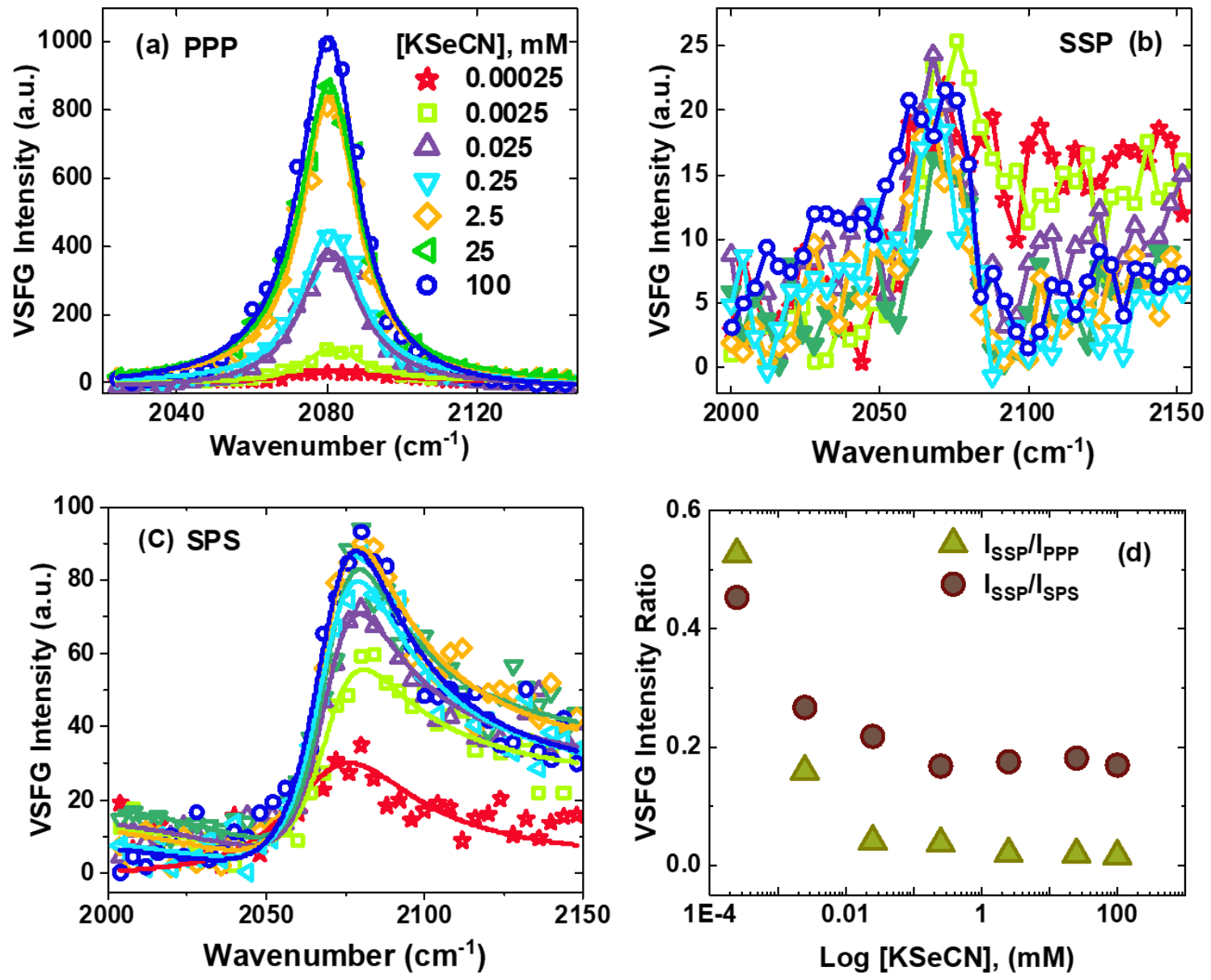
Figure 3. The variation of VSFG intensity for $\mathrm{CN}$ stretch region of $\mathrm{SeCN}^{-}$ions under (a) PPP, (b) SSP, and (c) SPS polarization combinations. The solid lines in (a) and (c) are the fits to the experimental data and in (b) are the lines following the data points as visual guides. Panel (d) is the variation of the ratio of the VSFG intensity obtained under different polarizations with KSeCN bulk concentration.

Relatively stronger VSFG signal under PPP polarization indicates a constructive interference between the $\chi^{(2)}$ components and $\mathrm{SeCN}^{-}$enrichment at the interface. The PPP polarization configuration probes the combination of zzz, xxz, zzx, and zxx components of $\chi^{(2)}$ while SSP and SPS selectively probes only xxz and xzx components, respectively. The intensity of VSFG signal is the product of effective number density (Ns) and the average orientation $(r(\theta))$ of $\mathrm{SeCN}^{-}$anions at interface as given by equation 3 . The SSP polarization probes the vibrational modes having a component of dipole moment perpendicular to the interface and the SPS detects those parallel to the interface. ${ }^{72}$ The lower intensity obtained under SSP and SPS polarization suggests that the $\mathrm{SeCN}^{-}$anions have intermediate orientation. By taking the ratio of the SSP to PPP and SSP to SPS signal, it is possible to determine the actual orientation of $\mathrm{SeCN}^{-}$anions. ${ }^{24,66}$ The corresponding SSP to PPP signal ratio varies from 0.6 to 0.02 , and the SSP to SPS signal ratio varies from 0.5 to 0.2 as the $\mathrm{KSeCN}$ concentration increases (Figure 3d). This suggests that the average orientation of anions with respect to surface normal ranges from $45^{\circ}$ to $22^{\circ}$ with the increasing $\mathrm{KSeCN}$ concentration. ${ }^{24}$ At lower concentrations, anions are loosely packed and orient at greater angle with respect to surface normal but with the increasing concentration, they orient more parallel to the surface normal. 


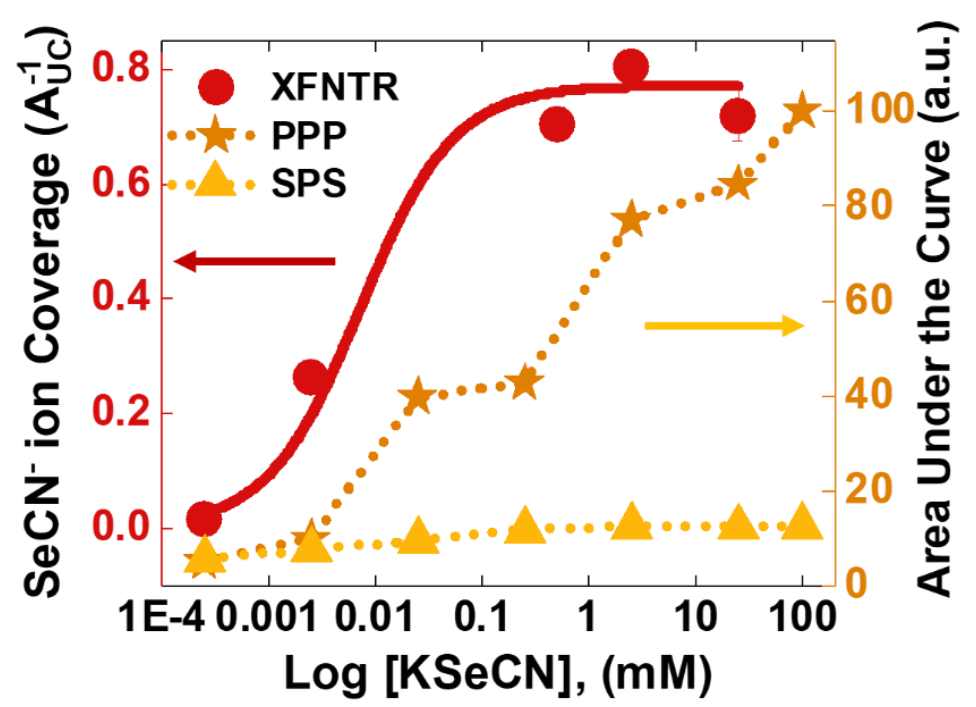

Figure 4. The variation of area under the CN stretch VSFG spectra (PPP and SPS polarizations) and the surface coverage of $\mathrm{SeCN}^{-}$ions as a function of $\mathrm{KSeCN}$ salt concentration obtained from XFNTR measurement. The red solid line in is the Langmuir fit to the corresponding XFNTR data set. The dotted lines are following the data points for clarity.

The results obtained from the analysis of XFNTR, and CN stretch of VSFG measurements are plotted together in Figure 4. Red circles are the average surface coverage of $\mathrm{SeCN}^{-}$anions obtained from XFNTR fit. Stars and triangles are the area under the curve of CN stretch region obtained from VSFG measurements under PPP and SPS polarizations, respectively, as a function of bulk KSeCN concentrations. The area under the curve is a function of the anion population and orientation (equation 3). A Langmuir adsorption isotherm is used to fit the $\mathrm{SeCN}^{-}$ion coverage obtained from XFNTR to display the variation. The full coverage ( $1 \mathrm{AUC}_{\mathrm{UC}}^{-1}$, where AUC is the unit cell area of a DPTAP molecule) is defined as the coverage when there is one $\mathrm{SeCN}^{-}$anion per DPTAP molecule at the interface. The surface coverage plot shows that the adsorption of $\mathrm{SeCN}^{-}$ ions increases with increasing bulk concentration and appears to saturate at $0.5 \mathrm{mM}$. Conversely, 
the area under the curve of $\mathrm{CN}$ stretch region under PPP polarization does not appear to saturate. These two results together suggest that the orientational re-organization of $\mathrm{SeCN}^{-}$ions continue after their total number in Stern and diffuse layers saturate. The increase in the VSFG signal under PPP combination is not exactly monotonic but appears to have some steps, i.e. the intensity stays almost constant within certain bulk concentration ranges before jumping to a higher value. A similar behavior was observed with $\mathrm{SCN}^{-}$in our previous studies. ${ }^{22}$

We used XR to investigate the details of the interfacial re-organization after apparent saturation of the $\mathrm{SeCN}^{-}$coverage (Figure 5). XR is sensitive to the electron density gradient at the interface. ${ }^{4}$ Therefore, $\mathrm{SeCN}^{-}$ions adsorbing in Stern layer can be detected from their effects on the total electron density around the head group region of the DPTAP. However, $\mathrm{SeCN}^{-}$ions in diffuse layer that do not form a well-defined layer have much smaller effect on $\mathrm{XR} .{ }^{59}$ Since XFNTR is sensitive to all $\mathrm{SeCN}^{-}$ions in both Stern and diffuse layers, and $\mathrm{XR}$ is mostly sensitive to the $\mathrm{SeCN}^{-}$ ions in Stern layer; their comparison will elucidate the relative distribution of $\mathrm{SeCN}^{-}$ions between these layers.

Figure 5a shows the X-ray reflectivity intensity (R) from DPTAP monolayer on $2.5 \mu \mathrm{M}$, $2.5 \mathrm{mM}$, and $25 \mathrm{mM} \mathrm{KSeCN}$ concentrations normalized to the Fresnel reflectivity $\left(\mathrm{R}_{\mathrm{F}}\right)$ from an ideally flat interface. The solid lines are the best fits to the experimental data, using a box model. This model represents the interface as uniform electron density layers with error function interfaces (to account for the roughness) between them. Then, the calculated reflectivity is compared to the data and the fit parameters, including the length, electron density, and roughness are optimized using a least-squares fitting method (Table S2). ${ }^{73}$ Electron density profiles (EDPs) are plotted by using these fit parameters (Figure 5b). 

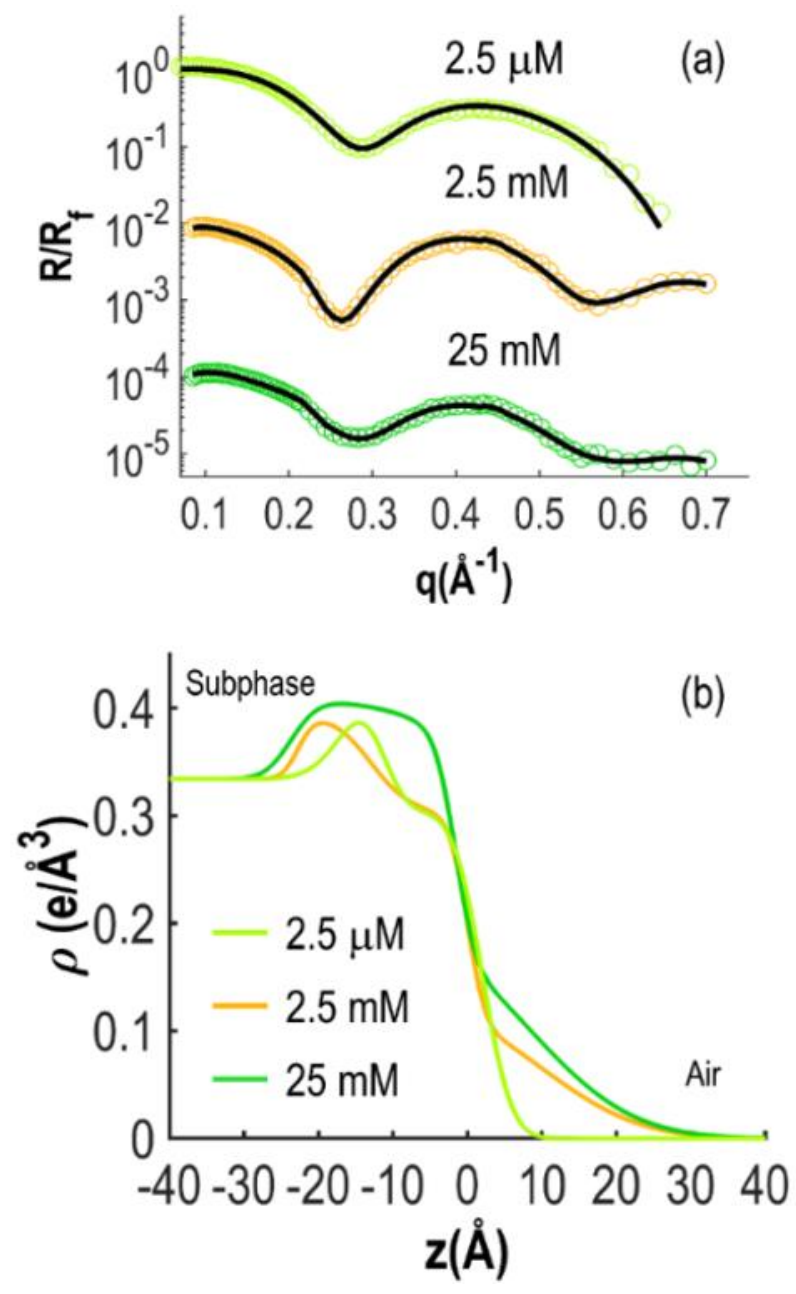

Figure 5. (a) XR data (symbols) and fit (lines) at three different bulk $\mathrm{KSeCN}$ concentrations. (b) EDPs derived from the fits to the XR data in (a).

The EDP of DPTAP on $2.5 \mu \mathrm{M} \mathrm{KSeCN}$ solution (Figure $5 \mathrm{~b}$ ) is very similar to that of on pure water which indicates negligible $\mathrm{SeCN}^{-}$adsorption in Stern layer. However, XFNTR shows $0.02 \mathrm{AUC}^{-1}$ coverage for $\mathrm{SeCN}^{-}$, which corresponds to $1 \mathrm{SeCN}^{-}$per $50 \mathrm{DPTAP}^{+}$. Similarly, VSFG shows small but detectable signal from $\mathrm{SeCN}^{-}$at all polarization combinations. As discussed above, the SSP/PPP and SPS/PPP intensity ratios suggest that the average angle from the surface normal is around $45^{\circ}$ at this concentration (Figure $3 \mathrm{~d}$ ), which is very similar to the orientation observed with $\mathrm{SCN}^{-}$at the free air/water interface. ${ }^{24}$ Therefore, it is reasonable to say that at 2.5 
$\mu \mathrm{M}$ bulk concentration, all the $\mathrm{SeCN}^{-}$ions adsorb in diffuse layer and they do not directly interact with the DPTAP monolayer.

At $2.5 \mathrm{mM}$ bulk concentration, the electron density at the headgroup region increases, suggesting some $\mathrm{SeCN}^{-}$adsorption in Stern layer. Also, the overall roughness of the interface increases. The increase in roughness is not specific to $\mathrm{SeCN}^{-}$. A similar trend was observed even with simpler anions, such as $\mathrm{Cl}^{-}, \mathrm{Br}^{-}$, and $\mathrm{I}^{-} .{ }^{53}$ According to the XFTNR, the total coverage of $\mathrm{SeCN}^{-}$is saturated at this concentration (Figure 2 and 4).

At $25 \mathrm{mM}$ bulk concentration, the electron density of the headgroup region significantly increases. Considering that the total $\mathrm{SeCN}^{-}$coverage determined by XFNTR has already saturated, it is reasonable to suggest that at this concentration, the ions that were already adsorbed in diffuse layer move to Stern layer, but the total coverage does not change. This behavior is very similar to the two-step adsorption observed with $\mathrm{PtCl}_{6}{ }^{2-}$ anions in previous studies. ${ }^{59,74}$ 

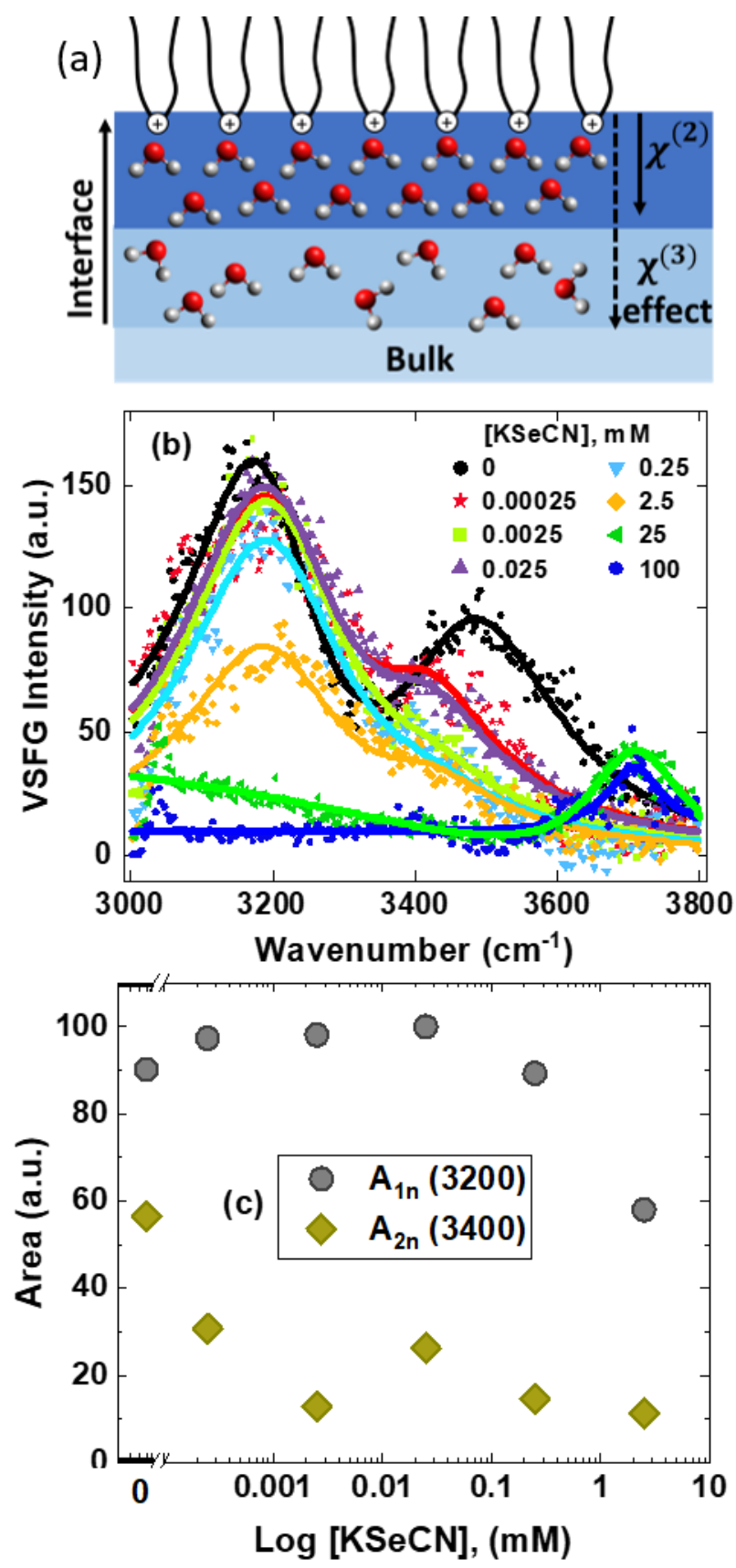

Figure 6. (a) Positively charged DPTAP ${ }^{+}$interface in absence of salt. (b) VSFG signal from the $\mathrm{OH}$-stretching region of water at various $\mathrm{KSeCN}$ concentrations under the DPTAP monolayer. The solid lines are the fit to the experimental data using two Lorentzian peaks (centered at $\sim 3180$ $\mathrm{cm}^{-1}$ and $\sim 3440 \mathrm{~cm}^{-1}$ ) as described in the text. (c) The variation of the area under each Lorentzian peak as a function of the bulk $\mathrm{KSeCN}$ concentration. 
The anion adsorption and its interfacial organization is further investigated by probing the $\mathrm{OH}$ stretch region of water under SSP polarization (Figure 6). The two peaks near $3200 \mathrm{~cm}^{-1}$ and $3400 \mathrm{~cm}^{-1}$ are characteristics of hydrogen bonded water molecules near the interface. Though the origins of these bands are still under debate, the bands at lower and higher wavenumber are assumed to originate from high degree and low degree of hydrogen bond ordering of the water molecules, respectively. ${ }^{75}, 76$ The $\mathrm{OH}$ stretch region is fitted globally with two-peak Lorentz function using equation 2. The peak positions and widths were forced to be same for all concentrations, varying only the amplitude of the resonances. The fit parameters obtained are tabulated in Table S3. The area under the curve for each Lorentz peak is calculated and plotted as a function of $\mathrm{KSeCN}$ concentration (Figure $6 \mathrm{c})$. The area under $\mathrm{A}_{1 \mathrm{n}}\left(3200 \mathrm{~cm}^{-1}\right)$ band, which originates due to the high degree of $\mathrm{H}$-bonding, remains mostly unchanged until the bulk $\mathrm{KSeCN}$ concentration reaches to $0.025 \mathrm{mM}$ and then drops at higher concentration. The band completely disappears for $25 \mathrm{mM}$ and $100 \mathrm{mM}$ salt concentrations. Conversely, the area under $\mathrm{A}_{2 \mathrm{n}}\left(3400 \mathrm{~cm}^{-1}\right)$ band which originates due to low degree of H-bonding sharply drops even for the lowest $\mathrm{KSeCN}$ concentration. With the increasing concentration, the $3400 \mathrm{~cm}^{-1}$ band either disappears or has very low intensity and only $3200^{-1} \mathrm{~cm}^{-1}$ band is visible. A qualitatively similar behavior was observed with $\mathrm{SCN}^{-}$ions in our previous studies. ${ }^{22}$ However, in that case, the $3400 \mathrm{~cm}^{-1}$ band disappeared at $0.025 \mathrm{mM}$ bulk concentration, while it is still visible at $2.5 \mathrm{mM}$ bulk concentration in this case. The overall line shape of $3200 \mathrm{~cm}^{-1}$ peak was also different with $\mathrm{SCN}^{-}$.

The charged interface induces strong H-bonding networks in water molecules which relax away in a few nanometers $(\sim 2 \mathrm{~nm})$, depending on the solution conditions,,${ }^{77}$ It is well known that the charged interface has second-order $\chi^{(2)}$ and third-order $\chi^{(3)}$ contribution to the VSFG signal. ${ }^{78}$ The $\chi^{(2)}$ contribution is from the interfacial molecules (primarily from Stern layer) which are 
asymmetrically oriented and hence induce an electric dipole allowed VSFG signal. The static electric field of the charged interface also aligns the bulk water molecules (in diffuse layer), which is called $\chi^{(3)}$ effect, where $\chi^{(3)}$ is the third order nonlinear susceptibility of water. So, the $\chi_{\text {eff }}^{(2)}$ has contributions from $\chi^{(2)}$ and $\chi^{(3)}$ effect which can be expressed as, $\chi_{e f f}^{(2)}=\chi^{(2)}+\chi^{(3)} \phi(0)$, where, $\phi(0)$ is the surface potential which decays as a function of distance from the interface. ${ }^{77}$, ${ }^{79-83} \mathrm{In}$ the absence of salt $(0 \mathrm{mM} \mathrm{KSeCN})$, the VSFG intensity of $\mathrm{OH}$ stretch region of water from the air/aqueous interface is strongly enhanced due to both $\chi^{(2)}$ and $\chi^{(3)}$ effects. This is schematically represented in Figure 6a. Addition of the salt decreases $\chi^{(2)}$ by breaking the interfacial H-bonding networks and eventually displacing water from the interface at higher salt concentrations. $\chi^{(3)}$ effect decreases after the addition of salt due to screening of static electric field of the charged interface.

At lower $\mathrm{KSeCN}$ concentration, $\chi^{(2)}$ remains unaffected, but the $\chi^{(3)}$ effect decreases due to screening of the static electric field of the charged interface experienced by the water molecules in diffuse layer. As a result, the weakly H-bonded water networks in diffused layer starts to break and the intensity of the $3400 \mathrm{~cm}^{-1}$ band decreases. ${ }^{84}$ This band is also shifted towards lower wavenumber region (red shift) which is the indication of increased interaction of water molecules with the surrounding ions, here $\mathrm{SeCN}^{-}{ }^{85}$ The $3200 \mathrm{~cm}^{-1}$ band remains almost unchanged until the $\mathrm{KSeCN}$ bulk concentration is $0.25 \mathrm{mM}$, suggesting that the interfacial H-bonding network (or $\left.\chi^{(2)}\right)$ is barely affected up to this concentration. The negligible Stern layer adsorption determined by XR at lower $\mathrm{KSeCN}$ concentration supports this interpretation (Figure 5b). We hypothesize that weakly hydrogen bonded water molecules are affected from the presence of small number of $\mathrm{SeCN}^{-}$ions but the strongly hydrogen bonded water molecules are not. Similar behavior was observed by Jena and Hore et al. at a charged silica interface under varying salt concentrations. ${ }^{79}$, 
${ }^{86}$ It is known that simple anions, such as $\mathrm{Cl}^{-},{ }^{53}$ or the anions that do not disturb the weak H-bonding network significantly, such as $\mathrm{NO}_{3}{ }^{-22},{ }^{22}$ lead to a more uniform decrease in VSFG signal mostly through the $\chi^{(3)}$ effect, without affecting the overall bimodal shape of the signal.

The $3200 \mathrm{~cm}^{-1}$ band decreases significantly (Figure 6c) as the concentration is increased from $0.25 \mathrm{mM}$ to $2.5 \mathrm{mM} \mathrm{KSeCN}$, which suggests that the ions start to move from diffuse to Stern layer. Consequently, the $\chi^{(2)}$ decreases due to breaking down of the strongly ordered interfacial H-bonding networks of water. The results obtained from the VSFG measurements of CN stretch region under PPP polarization also show a significant increase of its intensity (Figure 3a and 4) at this concentration window, referring to the possible transfer of ions and their reorganization. On the other hand, the slightly higher electron density profile at $2.5 \mathrm{mM} \mathrm{KSeCN}$ concentration compared to its value at lowest concentration (Figure 5b) suggests that the most of the $\mathrm{SeCN}^{-}$ions are still in diffuse layer.

At higher salt concentrations $(\geq 25 \mathrm{mM})$, the H-bonding environment of water near the interface is heavily affected by the adsorption of $\mathrm{SeCN}^{-}$anions and the $\mathrm{OH}$ stretch bands at 3200 $\mathrm{cm}^{-1}$ and $3400 \mathrm{~cm}^{-1}$ disappear. At this concentration, both $\chi^{(2)}$ and $\chi^{(3)}$ effects are significantly decreased due to the displacement of ordered interfacial water network by the $\mathrm{SeCN}^{-}$anions. This agrees with the XR data which shows that the electron density of the headgroup region significantly increases at $25 \mathrm{mM}$ bulk concentration, indicating enhanced Stern layer adsorption. Also, a new band around $3700 \mathrm{~cm}^{-1}$ appears. This band is commonly observed in the case of neat air/water interface and corresponds to the non-hydrogen bonded free $\mathrm{OH}$ that points towards the air. At air/water interface, more than $20 \%$ of water molecules have free non-hydrogen bonded $\mathrm{OH}$ which collectively give a sharp peak at $3700 \mathrm{~cm}^{-1}$. This peak is expected to disappear in the presence of DPTAP monolayer, as observed at lower salt concentrations $(\leq 2.5 \mathrm{mM})$. 
Reappearance of $3700 \mathrm{~cm}^{-1}$ peak at higher concentrations can be due to a few reasons. One possibility is that the monolayer structure is disturbed, and some parts of the surface is open, allowing free $\mathrm{OH}$ bonds to stick out of the surface. However, this possibility is ruled out by investigating the $\mathrm{CH}$ stretch region (Figure $\mathrm{S} 3$ ). The absence of any significant gauche defect from $\mathrm{CH}_{2}$ groups $\left(2855 \mathrm{~cm}^{-1}\right.$ band) for all concentrations indicates that the monolayer is well-packed and stable even at higher salt concentrations. Similarly, GID results show no difference between $2.5 \mathrm{mM}$ and $25 \mathrm{mM}$ samples (Figure $\mathrm{S} 2$ ). This is also supported by the monotonically increasing $\mathrm{CN}$ stretch intensity under PPP polarization (Figure $3 \mathrm{a}$ and 4), which could have decreased if the monolayer was largely disturbed and the overall surface charge density of DPTAP molecules decreased. There is slight decrease in $\mathrm{CH}$ peak intensity $\left(\mathrm{CH}_{3}\right.$ stretching at $2875 \mathrm{~cm}^{-1}$ and its Fermi resonance peak at $2937 \mathrm{~cm}^{-1}$ ) at higher concentration which is the evidence of subtle disorder in the monolayer packing, but this is unlikely to be enough to cause any open regions in the monolayer. In the presence of a stable and well-packed monolayer, it is unlikely that the reappearing $3700 \mathrm{~cm}^{-1}$ peak is solely a result of dangling $\mathrm{OH}$.

A second possibility is that the $3700 \mathrm{~cm}^{-1}$ is originating from the water molecules coordinating to $\mathrm{SeCN}^{-}$ions. It is found that the shape and phase of dangling $\mathrm{OH}$ and the $\mathrm{OH}$ mode of water complexed with anions are similar. ${ }^{84}$ This suggests that the origin of $3700 \mathrm{~cm}^{-1}$ peak at higher concentration could be the result of $\mathrm{SeCN}^{-}: \mathrm{H}_{2} \mathrm{O}$ complexation at Stern layer. Because, both XR (Figure 5b) and VSFG (Figure S3) do not show a significant difference in the tail region between $2.5 \mathrm{mM}$ and $25 \mathrm{mM}$ samples. The broadening of this peak compared to pure air/water interface indicates the presence of asymmetric bonding environments. Further investigations with molecular dynamics simulations can elucidate the origins of this peak as it was done in the case of $3600 \mathrm{~cm}^{-1}$ peak appearing in the presence of $\mathrm{PtCl}_{6}{ }^{2-}$ and $\mathrm{PdCl}_{4}{ }^{2-}$ anions. ${ }^{9,}{ }^{63}$ Nevertheless, VSFG 
studies of the $\mathrm{OH}$ stretch clearly show that the interfacial water reorganization continues above 0.1 mM bulk concentration.

Figure 7 summarizes the interpretation obtained by comparing all experimental data presented above. The change in interfacial water structure due to the adsorption of $\mathrm{SeCN}^{-}$ions as a function of $\mathrm{KSeCN}$ bulk concentration is demonstrated schematically by considering the three concentrations where the notable changes are observed. At very low concentration, $0.00025 \mathrm{mM}$, $\mathrm{SeCN}^{-}$ions are present only in diffuse layer without affecting Stern layer water structure. The total coverage is saturated around $0.5 \mathrm{mM}$ bulk concentration according to XFNTR result, and at 2.5 $\mathrm{mM}, \mathrm{SeCN}^{-}$ions start to move to Stern layer. At $25 \mathrm{mM}$, ions are significantly adsorbed at Stern layer which continue to reorganize until $100 \mathrm{mM}$ bulk concentration. These results highlight the importance of investigating the anion adsorption and the corresponding interfacial water structure for the better understanding of chemical, physical, and biological processes.

(a) $0.00025 \mathrm{mM}$

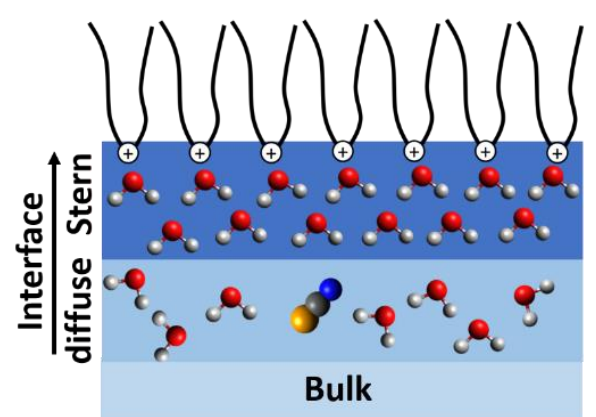

(b) $2.5 \mathrm{mM}$

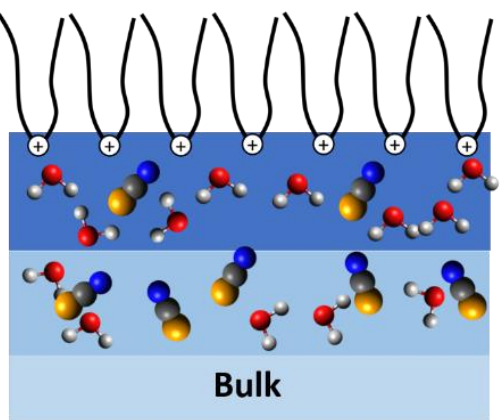

(c) $25 \mathrm{mM}$

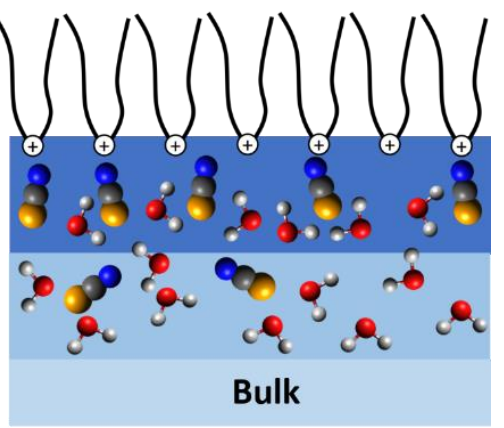

Figure 5. Schematic summarizing the results obtained from XFNTR, VSFG, and XR studies regarding the interfacial $\mathrm{SeCN}^{-}$and water organization as a function of bulk $\mathrm{KSeCN}$ concentration. (a) $0.00025 \mathrm{mM}$, ions are only in diffuse layer, (b) $2.5 \mathrm{mM}$, ions start to move to Stern layer but still majority of the ions are in diffuse layer, and (c) $25 \mathrm{mM} \mathrm{KSeCN}$, ions are now mostly in Stern layer. 


\section{CONCLUSIONS}

Complex anions, such as $\mathrm{SCN}^{-}$and $\mathrm{NO}_{3}^{-}$, play important roles in chemical separations and other environmental and industrial processes, yet very little is known about their interfacial effects. We have studied $\mathrm{SeCN}^{-}$adsorption at a positively charged DPTAP monolayer with multiple surface sensitive probes and identified important differences in the way they adsorb, compared to the simple anions, such as $\mathrm{Cl}^{-}$or $\mathrm{Br}^{-}$. At very low bulk concentrations $(<2.5 \mu \mathrm{M}), \mathrm{SeCN}^{-}$anions adsorb in diffuse layer only, without a direct interaction with the DPTAP. This is in contrast with the typical picture of ion adsorption at a charged interface, where the ions adsorb in Stern layer first. At intermediate bulk concentrations $(\sim 2.5 \mathrm{mM}), \mathrm{SeCN}^{-}$anion surface coverage reaches a maximum and the anions adsorb in both diffuse and Stern layers. They mostly disturb the weaklyhydrogen-bonded water molecules $\left(3400 \mathrm{~cm}^{-1}\right.$ region) but do not affect strongly-hydrogen-bonded water molecules $\left(3200 \mathrm{~cm}^{-1}\right.$ region). At the higher bulk concentrations $(>25 \mathrm{mM})$, the total $\mathrm{SeCN}^{-}$ surface coverage does not increase, but the majority of the anions move to Stern layer, their orientation become more parallel to the surface normal, typical water peaks (3200 and $\left.3400 \mathrm{~cm}^{-1}\right)$ disappear, and a new water population appears creating a VSFG signal at $3700 \mathrm{~cm}^{-1}$. These detailed observations were possible thanks to the complementary use of XFNTR, XR, and VSFG techniques which provided a unique sensitivity to the ions in diffuse and Stern layers as well as their orientational ordering. It is usually difficult to disentangle the effects of the number density and the orientational ordering on the VSFG signal intensity. Obtaining number density from XFNTR independently allowed a better interpretation of $-\mathrm{CN}$ stretch results. Utilizing XR to determine the ion adsorption in Stern layer helped in interpretation of the concentration dependent changes in $\mathrm{CN}$ and $\mathrm{OH}$ region VSFG experiments. These results clearly show that a simple quantitative list of strongly or weakly adsorbing ions (usually considered in the context of 
Hofmeister series) is not enough to describe the ion-specific effects of complex anions. Their interfacial adsorption behavior and effects on the hydrogen bonding network change significantly as a function of the surface coverage, which in turn, expected to affect the resulting interfacial processes in the presence of these complex anions.

ASSOCIATED CONTENT

Supporting Information. Fit parameters of $\mathrm{CN}$ and $\mathrm{OH}$ stretch region of VSFG spectra, and XR measurements; XFNTR plot at $25 \mathrm{mM} \mathrm{KSeCN}$ bulk concentration in absence of DPTAP monolayer; GID results, and CH stretch region of DPTAP monolayer under SSP polarization in presence of different $\mathrm{KSeCN}$ concentration. (PDF)

AUTHOR INFORMATION

\section{Corresponding Author}

*E-mail: ahmet@anl.gov. Web: http://www.anl.gov/profile/ahmet-uysal.com. Phone: +1-630252-9133.

\section{ACKNOWLEDGMENT}

This material is based upon work supported by the U.S. Department of Energy, Office of Science, Office of Basic Energy Sciences, Heavy Element Chemistry program under contract DE-AC0206CH11357. Use of the Advanced Photon Source, an Office of Science User Facility operated for the U.S. Department of Energy (DOE) Office of Science by Argonne National Laboratory, was supported by the U.S. DOE under Contract No. DE-AC02-06CH11357. NSF's ChemMatCARS Sector 15 is supported by the Divisions of Chemistry (CHE) and Materials Research (DMR), National Science Foundation, under Grant NSF/CHE-1834750. 


\section{REFERENCES}

1. Lo Nostro, P.; Ninham, B. W., Hofmeister phenomena: an update on ion specificity in biology. Chem. Rev. 2012, 112, 2286-2322.

2. Lovering, K. A.; Bertram, A. K.; Chou, K. C., New information on the ion-identitydependent structure of Stern layer revealed by sum frequency generation vibrational spectroscopy. J. Phys. Chem. C 2016, 120, 18099-18104.

3. Nagata, Y.; Hama, T.; Backus, E. H.; Mezger, M.; Bonn, D.; Bonn, M.; Sazaki, G., The surface of ice under equilibrium and nonequilibrium conditions. Acc. Chem. Res. 2019, 52, 10061015.

4. Bera, M. K.; Bu, W.; Uysal, A., Liquid Surface X-Ray Scattering. In Physical Chemistry of Gas-Liquid Interfaces, Elsevier: 2018; pp 167-194.

5. Lu, H.; Huang, Y.-C.; Hunger, J.; Gebauer, D.; Cölfen, H.; Bonn, M., Role of Water in CaCO3 Biomineralization. J. Am. Chem. Soc. 2021, 143, 1758-1762.

6. Yan, E. C.; Fu, L.; Wang, Z.; Liu, W., Biological macromolecules at interfaces probed by chiral vibrational sum frequency generation spectroscopy. Chem. Rev. 2014, 114, 8471-8498.

7. Liang, Z.; Bu, W.; Schweighofer, K. J.; Walwark, D. J.; Harvey, J. S.; Hanlon, G. R.; Amoanu, D.; Erol, C.; Benjamin, I.; Schlossman, M. L., Nanoscale view of assisted ion transport across the liquid-liquid interface. Proc. Natl. Acad. Sci. U. S. A. 2019, 116, 18227-18232.

8. Chowdhury, A. U.; Lin, L.; Doughty, B., Hydrogen-Bond-Driven Chemical Separations: Elucidating the Interfacial Steps of Self-Assembly in Solvent Extraction. ACS Appl. Mater. Interfaces 2020, 12, 32119-32130. 
9. Nayak, S.; Kumal, R. R.; Liu, Z.; Qiao, B.; Clark, A.; Uysal, A., Origins of Clustering of Metalate-Extractant Complexes in Liquid-Liquid Extraction. ACS Appl. Mater. Interfaces 2021, 13, 24194-24206.

10. Miller, M.; Liang, Y.; Li, H.; Chu, M.; Yoo, S.; Bu, W.; De La Cruz, M. O.; Dutta, P., Electrostatic origin of element selectivity during rare earth adsorption. Phys. Rev. Lett. 2019, 122, 058001-058001.

11. Kusaka, R.; Watanabe, M., Mechanism of phase transfer of uranyl ions: a vibrational sum frequency generation spectroscopy study on solvent extraction in nuclear reprocessing. Phys. Chem. Chem. Phys. 2018, 20, 29588-29590.

12. Scoppola, E.; Watkins, E. B.; Campbell, R. A.; Konovalov, O.; Girard, L.; Dufrêche, J. F.; Ferru, G.; Fragneto, G.; Diat, O., Solvent extraction: Structure of the liquid-liquid interface containing a diamide ligand. Angew. Chem. 2016, 128, 9472-9476.

13. Sun, P.; Huang, K.; Liu, H., Specific Salt Effect on the Interaction between Rare Earth Ions and Trioctylphosphine Oxide Molecules at the Organic-Aqueous Two-Phase Interface: Experiments and Molecular Dynamics Simulations. Langmuir 2018, 34, 11374-11383.

14. Fenter, P.; Lee, S. S., Hydration layer structure at solid-water interfaces. MRS Bull. 2014, 39, 1056-1061.

15. Johnson, C. M.; Baldelli, S., Vibrational sum frequency spectroscopy studies of the influence of solutes and phospholipids at vapor/water interfaces relevant to biological and environmental systems. Chem. Rev. 2014, 114, 8416-8446.

16. Grooms, A. J.; Neal, J. F.; Ng, K. C.; Zhao, W.; Flood, A. H.; Allen, H. C., Thermodynamic Signatures of the Origin of Anti-Hofmeister Selectivity for Phosphate at Aqueous Interfaces. J. Phys. Chem. A 2020, 124, 5621-5630. 
17. Fumagalli, L.; Esfandiar, A.; Fabregas, R.; Hu, S.; Ares, P.; Janardanan, A.; Yang, Q.; Radha, B.; Taniguchi, T.; Watanabe, K., Anomalously low dielectric constant of confined water. Science 2018, 360, 1339-1342.

18. Bera, M. K.; Antonio, M. R., Polynuclear Speciation of Trivalent Cations near the Surface of an Electrolyte Solution. Langmuir 2015, 31, 5432-5439.

19. Jungwirth, P.; Cremer, P. S., Beyond hofmeister. Nat. Chem. 2014, 6, 261-263.

20. Leontidis, E., Investigations of the Hofmeister series and other specific ion effects using lipid model systems. Adv. Colloid Interface Sci. 2017, 243, 8-22.

21. Kumal, R. R.; Nguyenhuu, H.; Winter, J. E.; McCarley, R. L.; Haber, L. H., Impacts of salt, buffer, and lipid nature on molecular adsorption and transport in liposomes as observed by second harmonic generation. J. Phys. Chem. C 2017, 121, 15851-15860.

22. Lovering, K.; Nayak, S.; Bu, W.; Uysal, A., The Role of Specific Ion Effects in Ion Transport: The Case of Nitrate and Thiocyanate. J. Phys. Chem. C 2019, 124, 573-581.

23. Millefiori, S.; Foffani, A., Dipole moments and rotational isomerism for organic thio-and selenocyanates. Tetrahedron 1966, 22, 803-811.

24. Viswanath, P.; Motschmann, H., Oriented thiocyanate anions at the air- electrolyte interface and its implications on interfacial water-a vibrational sum frequency spectroscopy study. J. Phys. Chem. C 2007, 111, 4484-4486.

25. Tesei, G.; Aspelin, V.; Lund, M., Specific Cation Effects on SCN-in Bulk Solution and at the Air-Water Interface. J. Phys. Chem. B 2018, 122, 5094-5105.

26. Viswanath, P.; Aroti, A.; Motschmann, H.; Leontidis, E., Vibrational Sum Frequency Generation Spectroscopic Investigation of the Interaction of Thiocyanate Ions with Zwitterionic Phospholipid Monolayers at the Air- Water Interface. J. Phys. Chem. B 2009, 113, 14816-14823. 
27. Viswanath, P.; Motschmann, H., Effect of interfacial presence of oriented thiocyanate on water structure. J. Phys. Chem. C 2008, 112, 2099-2103.

28. Hao, H.; Xie, Q.; Ai, J.; Wang, Y.; Bian, H., Specific counter-cation effect on the molecular orientation of thiocyanate anions at the aqueous solution interface. Phys. Chem. Chem. Phys. 2020, 22, 10106-10115.

29. Mizuno, H.; Rizzuto, A. M.; Saykally, R. J., Charge-Transfer-to-Solvent Spectrum of Thiocyanate at the Air/Water Interface Measured by Broadband Deep Ultraviolet Electronic Sum Frequency Generation Spectroscopy. J. Phys. Chem. Lett. 2018, 9, 4753-4757.

30. Onorato, R. M.; Otten, D. E.; Saykally, R. J., Adsorption of thiocyanate ions to the dodecanol/water interface characterized by UV second harmonic generation. Proc. Natl. Acad. Sci. U. S. A. 2009, 106, 15176-15180.

31. Schultz, P. W.; Leroi, G. E.; Popov, A. I., Solvation of SCN-and SeCN-anions in hydrogen-bonding solvents. J. Am. Chem. Soc. 1996, 118, 10617-10625.

32. Lenchenkov, V.; She, C.; Lian, T., Vibrational relaxation of cn stretch of pseudo-halide anions (OCN-, SCN-, and SeCN-) in polar solvents. J. Phys. Chem. B 2006, 110, 19990-19997.

33. Yuan, R.; Yan, C.; Tamimi, A.; Fayer, M. D., Molecular anion hydrogen bonding dynamics in aqueous solution. J. Phys. Chem. B 2015, 119, 13407-13415.

34. Lindquist, B. A.; Corcelli, S. A., Nitrile Groups as Vibrational Probes: Calculations of the $\mathrm{C} \square \mathrm{N}$ Infrared Absorption Line Shape of Acetonitrile in Water and Tetrahydrofuran. J. Phys. Chem. B 2008, 112, 6301-6303.

35. Karmakar, A.; Duvail, M.; Bley, M.; Zemb, T.; Dufrêche, J.-F., Combined supramolecular and mesoscale modelling of liquid-liquid extraction of rare earth salts. Colloids Surf., A 2018, 555, 713-727. 
36. Preez, A. d.; S Preston, J., The solvent extraction of rare-earth metals by carboxylic acids. Solvent Extr. Ion Exch. 1992, 10, 207-230.

37. Nayak, S.; Lovering, K. A.; Bu, W.; Uysal, A., Anions Enhance Rare Earth Adsorption at Negatively Charged Surfaces. J. Phys. Chem. Lett. 2020, 11, 4436-4442.

38. Nayak, S.; Lovering, K.; Uysal, A., Ion-Specific Clustering of Metal-Amphiphile Complexes in Rare Earth Separations. Nanoscale 2020, 12, 20202-20210.

39. Gradzielski, M.; Duvail, M.; de Molina, P. M.; Simon, M.; Talmon, Y.; Zemb, T., Using Microemulsions: Formulation Based on Knowledge of Their Mesostructure. Chem. Rev. 2021, $121,5671-5740$.

40. Wang, J.; Arrachart, G.; Giusti, F.; Martin-Gassin, G.; Gassin, P.-M.; Jonchere, A.; Diat, O.; Girard, L., Synthesis and Characterization of a Chromo-Extractant to the Probe LiquidLiquid Interface in a Solvent Extraction Process. J. Phys. Chem. C 2020, 124, 10916-10923.

41. Chen, X.; Hua, W.; Huang, Z.; Allen, H. C., Interfacial water structure associated with phospholipid membranes studied by phase-sensitive vibrational sum frequency generation spectroscopy. J. Am. Chem. Soc. 2010, 132, 11336-11342.

42. Mondal, J. A.; Nihonyanagi, S.; Yamaguchi, S.; Tahara, T., Three distinct water structures at a zwitterionic lipid/water interface revealed by heterodyne-detected vibrational sum frequency generation. J. Am. Chem. Soc. 2012, 134, 7842-7850.

43. Sthoer, A.; Tyrode, E., Interactions of $\mathrm{Na}+$ cations with a highly charged fatty acid langmuir monolayer: molecular description of the phase transition. J. Phys. Chem. C 2019, 123, 23037-23048.

44. Sung, W.; Krem, S.; Kim, D., Binding of trivalent ions on fatty acid Langmuir monolayer: Fe3+ versus La3+. J. Chem. Phys. 2018, 149, 163304-163307. 
45. Yan, C.; Thomaz, J. E.; Wang, Y.-L.; Nishida, J.; Yuan, R.; Breen, J. P.; Fayer, M. D., Ultrafast to ultraslow dynamics of a Langmuir monolayer at the air/water interface observed with reflection enhanced 2D IR spectroscopy. J. Am. Chem. Soc. 2017, 139, 16518-16527.

46. Uysal, A.; Chu, M.; Stripe, B.; Timalsina, A.; Chattopadhyay, S.; Schlepütz, C. M.; Marks, T. J.; Dutta, P., What $\mathrm{x}$ rays can tell us about the interfacial profile of water near hydrophobic surfaces. Phys. Rev. B 2013, 88, 035431-035438.

47. Zdrali, E.; Okur, H. I.; Roke, S., Specific ion effects at the interface of nanometer-sized droplets in water: structure and stability. J. Phys. Chem. C 2019, 123, 16621-16630.

48. Hamal, P.; Nguyenhuu, H.; Subasinghege Don, V.; Kumal, R. R.; Kumar, R.; McCarley, R. L.; Haber, L. H., Molecular adsorption and transport at liposome surfaces studied by molecular dynamics simulations and second harmonic generation spectroscopy. J. Phys. Chem. B 2019, 123, $7722-7730$.

49. Hamal, P., Second Harmonic Generation Spectroscopy and Microscopy of Liposomes, Nanoparticles, and Cells. 2020.

50. Eisenthal, K., Liquid interfaces probed by second-harmonic and sum-frequency spectroscopy. Chem. Rev. 1996, 96, 1343-1360.

51. Geiger, F. M., Second harmonic generation, sum frequency generation, and $\chi$ (3): Dissecting environmental interfaces with a nonlinear optical Swiss Army knife. Annu. Rev. Phys. Chem. 2009, 60, 61-83.

52. Horowitz, Y.; Steinruck, H.-G.; Han, H.-L.; Cao, C.; Abate, I. I.; Tsao, Y.; Toney, M. F.; Somorjai, G. A., Fluoroethylene carbonate induces ordered electrolyte interface on silicon and sapphire surfaces as revealed by sum frequency generation vibrational spectroscopy and X-ray reflectivity. Nano Lett. 2018, 18, 2105-2111. 
53. Sung, W.; Wang, W.; Lee, J.; Vaknin, D.; Kim, D., Specificity and variation of length scale over which monovalent halide ions neutralize a charged interface. J. Phys. Chem. C 2015, $119,7130-7137$.

54. Nojima, Y.; Suzuki, Y.; Yamaguchi, S., Weakly hydrogen-bonded water inside charged lipid monolayer observed with heterodyne-detected vibrational sum frequency generation spectroscopy. J. Phys. Chem. C 2017, 121, 2173-2180.

55. Mondal, J. A.; Nihonyanagi, S.; Yamaguchi, S.; Tahara, T., Structure and orientation of water at charged lipid monolayer/water interfaces probed by heterodyne-detected vibrational sum frequency generation spectroscopy. J. Am. Chem. Soc. 2010, 132, 10656-10657.

56. Krem, S.; Lee, M.; Sam, S.; Sung, W.; Kim, D., Structure of Electric Double Layer under Cationic Langmuir Monolayer: Charge Condensation Effect. J. Phys. Chem. Lett. 2021, 12, 34173423.

57. Sung, W.; Kim, D.; Shen, Y., Sum-frequency vibrational spectroscopic studies of Langmuir monolayers. Curr. Appl. Phys. 2013, 13, 619-632.

58. Rao, Y.; Tao, Y.-s.; Wang, H.-f., Quantitative analysis of orientational order in the molecular monolayer by surface second harmonic generation. J. Chem. Phys. 2003, 119, 52265236.

59. Uysal, A.; Rock, W.; Qiao, B.; Bu, W.; Lin, B., Two-Step Adsorption of PtCl62Complexes at a Charged Langmuir Monolayer: Role of Hydration and Ion Correlations. J. Phys. Chem. C 2017, 121, 25377-25383.

60. Bu, W.; Vaknin, D., X-ray fluorescence spectroscopy from ions at charged vapor/water interfaces. J. Appl. Phys. 2009, 105 (8), 084911. 
61. Bu, W.; Hou, B.; Mihaylov, M.; Kuzmenko, I.; Lin, B.; Meron, M.; Soderholm, L.; Luo, G.; Schlossman, M. L., X-ray fluorescence from a model liquid/liquid solvent extraction system. J. Appl. Phys. 2011, 110, 102214-102216.

62. Bu, W.; Mihaylov, M.; Amoanu, D.; Lin, B.; Meron, M.; Kuzmenko, I.; Soderholm, L.; Schlossman, M. L., X-ray studies of interfacial strontium-extractant complexes in a model solvent extraction system. J. Phys. Chem. B 2014, 118, 12486-12500.

63. Rock, W.; Qiao, B.; Zhou, T.; Clark, A. E.; Uysal, A., Heavy Anionic Complex Creates a Unique Water Structure at a Soft Charged Interface. J. Phys. Chem. C 2018, 122, 29228-29236.

64. Ishiyama, T.; Imamura, T.; Morita, A., Theoretical studies of structures and vibrational sum frequency generation spectra at aqueous interfaces. Chem. Rev. 2014, 114, 8447-8470.

65. Zhang, Z.; Kim, J.; Khoury, R.; Saghayezhian, M.; Haber, L. H.; Plummer, E., Surface sum frequency generation spectroscopy on non-centrosymmetric crystal GaAs (001). Surf. Sci. 2017, 664, 21-28.

66. Zhuang, X.; Miranda, P.; Kim, D.; Shen, Y., Mapping molecular orientation and conformation at interfaces by surface nonlinear optics. Phys. Rev. B 1999, 59, 12632-12640.

67. Verreault, D.; Hua, W.; Allen, H. C., From conventional to phase-sensitive vibrational sum frequency generation spectroscopy: probing water organization at aqueous interfaces. J. Phys. Chem. Lett. 2012, 3, 3012-3028.

68. Lambert, A. G.; Davies, P. B.; Neivandt, D. J., Implementing the theory of sum frequency generation vibrational spectroscopy: a tutorial review. Appl. Spectrosc. Rev. 2005, 40, 103-145.

69. Stiopkin, I. V.; Jayathilake, H. D.; Bordenyuk, A. N.; Benderskii, A. V., Heterodynedetected vibrational sum frequency generation spectroscopy. J. Am. Chem. Soc. 2008, 130, 22712275 . 
70. Málková, Šs; Long, F.; Stahelin, R. V.; Pingali, S. V.; Murray, D.; Cho, W.; Schlossman, M. L., X-ray reflectivity studies of cPLA2 $\alpha-\mathrm{C} 2$ domains adsorbed onto Langmuir monolayers of SOPC. Biophys. J. 2005, 89, 1861-1873.

71. Bu, W.; Yu, H.; Luo, G.; Bera, M. K.; Hou, B.; Schuman, A. W.; Lin, B.; Meron, M.; Kuzmenko, I.; Antonio, M. R., Observation of a rare earth ion-extractant complex arrested at the oil-water interface during solvent extraction. J. Phys. Chem. B 2014, 118, 10662-10674.

72. Altman, R. M.; Richmond, G. L., Coming to Order: Adsorption and Structure of Nonionic Polymer at the Oil/Water Interface as Influenced by Cationic and Anionic Surfactants. Langmuir 2020, 36, 1975-1984.

73. Danauskas, S. M.; Li, D.; Meron, M.; Lin, B.; Lee, K. Y. C., Stochastic fitting of specular X-ray reflectivity data using StochFit. J. Appl. Crystallogr. 2008, 41, 1187-1193.

74. Rock, W.; Oruc, M. E.; Ellis, R. J.; Uysal, A., Molecular scale description of anion competition on amine-functionalized surfaces. Langmuir 2016, 32, 11532-11539.

75. Gragson, D.; Richmond, G., Investigations of the structure and hydrogen bonding of water molecules at liquid surfaces by vibrational sum frequency spectroscopy. J. Phys. Chem. B 1998, $102(20), 3847-3861$.

76. Shen, Y. R.; Ostroverkhov, V., Sum-frequency vibrational spectroscopy on water interfaces: polar orientation of water molecules at interfaces. Chem. Rev. 2006, 106, 1140-1154.

77. Wen, Y.-C.; Zha, S.; Liu, X.; Yang, S.; Guo, P.; Shi, G.; Fang, H.; Shen, Y. R.; Tian, C., Unveiling microscopic structures of charged water interfaces by surface-specific vibrational spectroscopy. Phys. Rev. Lett. 2016, 116, 016101-016105. 
78. Yan, E. C.; Liu, Y.; Eisenthal, K. B., New method for determination of surface potential of microscopic particles by second harmonic generation. J. Phys. Chem. B 1998, 102 (33), 63316336.

79. Jena, K. C.; Covert, P. A.; Hore, D. K., The effect of salt on the water structure at a charged solid surface: Differentiating second-and third-order nonlinear contributions. J. Phys. Chem. Lett. 2011, 2, 1056-1061.

80. Kumal, R. R. Nanoparticle-Based Drug-Delivery Systems Studied by Second Harmonic Generation. Ph.D. Dissertation, Louisiana State University, Baton Rouge, LA, 2017.

81. Rehl, B.; Gibbs, J. M., Role of Ions on the Surface-Bound Water Structure at the Silica/Water Interface: Identifying the Spectral Signature of Stability. J. Phys. Chem. Lett. 2021, $12,2854-2864$.

82. Ohno, P. E.; Wang, H.-f.; Geiger, F. M., Second-order spectral lineshapes from charged interfaces. Nat. Commun. 2017, 8, 1-9.

83. Kumal, R. R.; Karam, T. E.; Haber, L. H., Determination of the surface charge density of colloidal gold nanoparticles using second harmonic generation. J. Phys. Chem. C 2015, 119, 16200-16207.

84. Tarbuck, T. L.; Richmond, G. L., Adsorption and Reaction of CO2 and SO2 at a Water Surface. J. Am. Chem. Soc. 2006, 128, 3256-3267.

85. Allen, H. C.; Casillas-Ituarte, N. N.; Sierra-Hernandez, M. R.; Chen, X.; Tang, C. Y., Shedding light on water structure at air-aqueous interfaces: ions, lipids, and hydration. Phys. Chem. Chem. Phys. 2009, 11, 5538-5549.

86. Covert, P. A.; Jena, K. C.; Hore, D. K., Throwing salt into the mix: Altering interfacial water structure by electrolyte addition. J. Phys. Chem. Lett. 2014, 5, 143-148. 